\title{
Why are pathogenic SIV and HIV infections characterized by high level immune activation when other chronic virus infection are not? Lessons from nonpathogenic SIV infection in natural host species Silvija Staprans, Sara Klucking, Guido Silvestri, Rahul Chavan, Ashley Barry and Mark Feinberg*
}

Address: Emory Vaccine Center, Emory University, Atlanta, Georgia

* Corresponding author

from 2006 International Meeting of The Institute of Human Virology Baltimore, USA. |7-2| November, 2006

Published: 21 December 2006

Retrovirology 2006, 3(Suppl I):S47 doi:10.1 I86/1742-4690-3-SI-S47

(C) 2006 Staprans et al; licensee BioMed Central Ltd.

Pathogenic SIV infections of non-human primates (such as rhesus macaques [RMs]) and HIV infections of humans are characterized by progressive CD4 T cell depletion and dysfunction in association with high levels of virus replication and generalized immune activation. In marked contrast, non-pathogenic infections of non-human primate species that are natural hosts for SIV infection, such as sooty mangabeys (SMs), are characterized by the numerical and functional preservation of CD4 T cell populations and the absence of aberrant immune activation despite levels of virus replication that equal or exceed those commonly found in pathogenic SIV and HIV infections. In light of these observations, it appears that chronic generalized immune activation and its associated immunopathology are more important drivers of progression to AIDS than the direct cytopathic consequences of virus infection. To better understand the cellular and molecular bases of whether or not chronic immune activation and attendant immunopathology follow CD4+ T cell tropic lentivirus infection, we studied how and when host innate and adaptive immune responses diverge following SIV infection of SMs and RMs, and compared these results with those obtained in studies of HIV-infected humans. Interestingly, differences in innate immune responses (eg, dendritic cell activation and migration) to infection are evident from the first days of infection, which are then followed by substantive differences in the magnitude and nature of adaptive immune responses. These differences in in vivo behavior of dendritic cells are recapitulated following ex vivo exposure of SM, RM and human plasmacytoid dendritic cells to specific TLR ligands and to inactivated virus. In contrast to pDCs from RMs and humans, pDCs from SMs fail to produce IFNalpha following exposure to specific TLR ligands and to SIV - apparently the result of divergent propagation of activation signals along post-receptor pathways. At the organismal level, gene expression profiling studies further indicate that a major feature distinguishing pathogenic from non-pathogenic CD4+ T cell tropic lentivirus infections is the extent to which a pattern of type 1 interferon production and response profiles are manifest. In all, our results indicate that natural host species, such as SMs, may avoid AIDS by the genetically programmed failure of their innate immune systems to respond to the presence of virus in a way that initiates and maintains a state of generalized and deleterious immune activation. 